\title{
Effloreszenzen und Blutbildveränderungen bei einem 9 Monate alten Säugling mit Glutarazidurie Typ I
}

\author{
E. Landmann², J. Kreuder ${ }^{3}$, L. Gortner ${ }^{2}$, M. Neugebauer ${ }^{1}$, A. Reiter ${ }^{1}$, R. Blütters-Sawatzki ${ }^{1}$ \\ 1 Zentrum für Kinderheilkunde, Abt. für Hämatologie und Onkologie, Justus-Liebig-Universität, Gießen \\ 2 Zentrum für Kinderheilkunde, Abt. für Allgemeine Pädiatrie und Neonatologie, Justus-Liebig-Universität, Gießen \\ ${ }^{3}$ Zentrum für Kinderheilkunde, Abt. für Kardiologie, Justus-Liebig-Universität, Gießen
}

\section{Zusammenfassung}

Unspezifische Hautauffälligkeiten kommen bei bis zu $50 \%$ der an einer akuten myeloischen Leukämie erkrankten Patienten vor. Bei den myelomonoblastären Formen treten in etwa $30 \%$ der Fälle Hautinfiltrate mit bioptisch nachweisbaren Tumorzellen auf. Diese können dem systemischen Auftreten maligner Blasten vorausgehen. Wir berichten über eine 9 Monate alte Patientin mit Glutarazidurie Typ I, die an einer AML (FAB M5) erkrankte. Zu den initialen Symptomen zählten leukämische Hautinfiltrate, eine Anämie und eine Leukopenie.

Cutaneous lesions and blood count changes in a 9-month-old girl with glutaric aciduria type I

Non-specific cutaneous lesions are common in patients suffering from acute myeloid leukemia (AML). Leukemic skin infiltrates are present in about $30 \%$ of cases of monoblastic or myelomonocytic leukemia. The appearance of specific skin lesions can precede bone marrow involvement. We report the case of a 9-month-old girl with acute myelogenous leukemia (FAB M5) and glutaric aciduria type I which initially presented with cutaneous lesions, anemia and leukopenia.

\section{Anamnese}

Bei der 9 Monate alten Patientin wurde im Alter von 4 Monaten eine Glutarazidurie Typ I diagnostiziert. Unter lysinarmer und tryptophanreduzierter Diät sowie L-Carnitin-Substitution waren die Stoffwechseleinstellung und die neurologische Entwicklung des Kindes zufriedenstellend.

Aktuell erfolgte die stationäre Einweisung wegen seit 10 Tagen bestehenden Hustens, intermittierenden Erbrechens und reduzierter Nahrungsaufnahme sowie ausgeprägter Anämie.

\section{Untersuchungsbefund}

Neun Monate alter weiblicher Säugling in leicht reduziertem Allgemeinzustand, Gewicht $7280 \mathrm{~g}$ (P 25), Länge $73 \mathrm{~cm}$ (P 75), Kopfumfang 46,5 cm (P 90-97). Körpertemperatur $38,1^{\circ} \mathrm{C}$ rektal. Die Schädelnähte waren klaffend, die Fontanelle tastete sich weich und im Niveau. Am rechten Unterbauch waren bläuliche derbe Papeln, 0,5-1 cm im Durchmesser messend, sichtbar (Abb.1). Rachenring leicht gerötet, Atemfrequenz 50/min, grob-

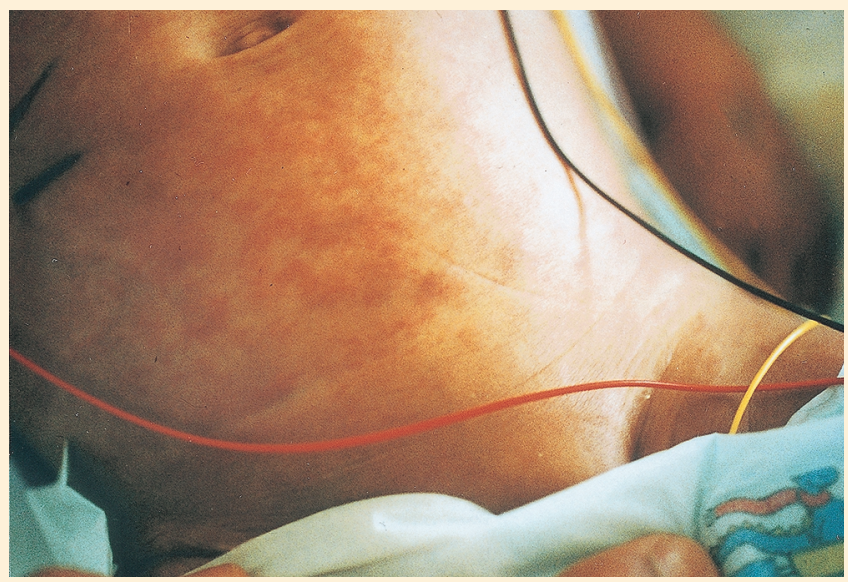

Abb. 1 Hautbefund bei Aufnahme: Bläuliche derbe Papeln bei gleichzeitig bestehendem Arzneimittelexanthem. blasige Rasselgeräusche ubiquitär, Herzfrequenz 160/min, Leber $1 \mathrm{~cm}$ unter dem Rippenbogen palpabel, Milz nicht palpabel. Instabilität und deutliche muskuläre Hypotonie des Rumpfes mit dyston-dyskinetischen Bewegungen beim Greifen. Keine Lymphadenopathie.

\section{Labor und technische Untersuchungen}

Blutbild: Leukozyten 4,9G/I (Granulozyten 0G/l, Lymphozyten 4,6 G/I, lymph. Reizformen 0,2 G/I, Monozyten 0,05 G/I, Eosinophile $0 \mathrm{G} / \mathrm{I}$ ), Erythrozyten 2,4 T/I, Hämoglobin $6,4 \mathrm{~g} / \mathrm{dl}$, Hämatokrit $18 \%$, MCV 76 fl, MCH 27 pg, Retikulozyten 1 0/00, Thrombozyten $255 \mathrm{G} / \mathrm{l}$

Weitere Laborbefunde: CRP 6,8 mg/l, im Normbereich lagen u.a. Transaminasen, LDH, Ferritin, Transferrin, Laktat, Ammoniak, Pyruvat, Carnitin. Bei Aufnahme Glutar- und Hydroxyglutarsäure im Urin geringfügig erhöht, bei Kontrolle am vierten stationären Tag im Normbereich.

\section{Therapie und Verlauf}

Eine Pneumonie wurde radiologisch ausgeschlossen. Wir führten die bereits ambulant initiierte orale Erythromycintherapie und Mukolyse fort. Zur Vermeidung einer katabolen Stoffwechsellage erhielt das Kind Infusionen mit hohem Glukoseanteil.

Unter dieser Therapie blieb die klinische Symptomatik zunächst unverändert und die Stoffwechseleinstellung stabil. Es persistierte eine ausgeprägte normozytäre, normochrome hyporegeneratorische Anämie (minimaler Hämoglobinwert 5,3g/dl) und die Leukozytenzahlen waren anhaltend niedrig (4200-6500 Leukozyten/ $\mu$ I mit Neutropenie).

Fünf Tage nach Aufnahme kam es zu einer deutlichen Verschlechterung des Allgemeinzustandes mit hochfieberhaften Temperaturen, Lebervergrößerung und Zunahme des Exanthems im Bereich des Bauchs bei unklarem Infektfokus. Laborchemisch fielen ein nur diskret erhöhter CRP-Wert (8,2 mg/l) und ein Anstieg der LDH auf $534 \mathrm{U} / \mathrm{l}$ auf. Im Knochenmarkpunktat fanden sich $94 \%$ undifferenzierte Blasten. 


\section{Diagnose}

Leukämische Hautinfiltrate bei akuter monoblastärer Leukämie (FAB: M 5; monoklonaler Antikörper 7.1 positiv).

\section{Kommentar}

Hautauffälligkeiten werden bei bis zu $50 \%$ der an einer akuten myeloischen Leukämie erkrankten Patienten beschrieben $[1,2]$. Jedoch bei nur 2-8\% der AML-Patienten treten Hautinfiltrate mit bioptisch nachweisbaren Tumorzellen auf [2,3]. In der Untergruppe der monozytären und myelomonozytären Formen sind hingegen bis zu $30 \%$ der Erkrankten betroffen [4, 5].

Meist sind derbe bläuliche Infiltrate ohne bevorzugte Lokalisation zu sehen. Diese können auch generalisiert oder in seltenen Fällen isoliert auftreten. Chlorome treten v.a. bei myeloischen differenzierten Leukämien auf. Sie erscheinen durch die Myeloperoxidase grünlich und finden sich bevorzugt an der Kopfhaut und periorbital (Abb. 2). Leukämische Infiltrate der Haut können dem Erscheinen von Blasten im Knochenmark oder im peripheren Blutbild um Wochen bis Monate vorausgehen [6, 7].

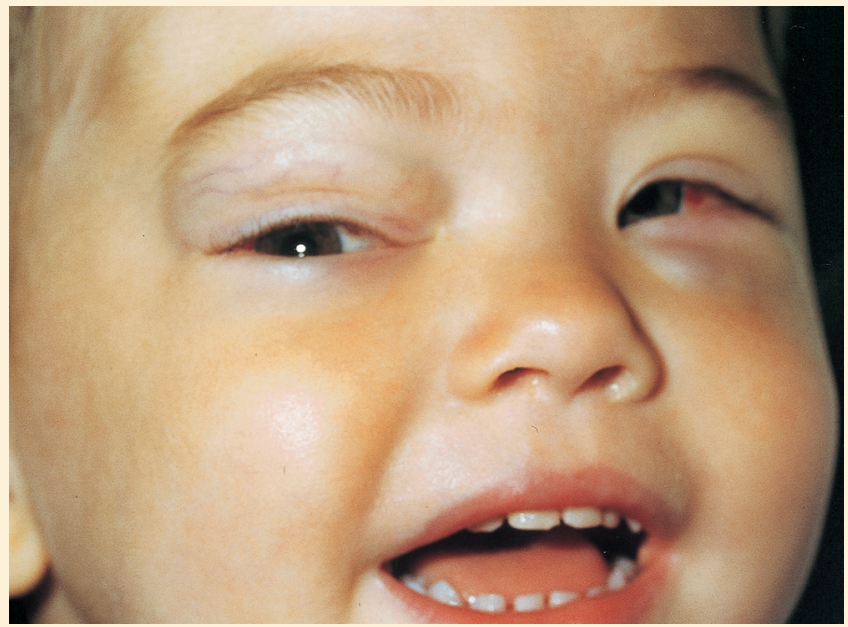

Abb. 2 Periorbitale und die Tränendrüse infiltrierende leukämische Infiltrate bei einem Patienten mit AML (M 5 n. FAB).

Differenzialdiagnostisch kommen in erster Linie Hautmetastasen eines Neuroblastoms und kutane Mastozytosen infrage. Mittels histologischer Untersuchungen können diese Erkrankungen von leukämischen Infiltraten abgegrenzt werden. Im Falle leukämischer Infiltrate sind im Hautbiopsat Blasten nachweisbar (Abb. 3). Das histologische Bild der leukämischen Infiltrate entspricht somit dem Bild bei Knochenmarkbefall (vergleiche Abb. 3 u. 4).

Eine histologische Untersuchung unklarer Effloreszenzen bei gleichzeitig bestehenden Blutbildveränderungen sollte daher frühzeitig in Erwägung gezogen werden.

Die Erkrankung an einer akuten monoblastären Leukämie bei gleichzeitig bestehender Glutarazidurie Typ I ist nach unserem Kenntnisstand bisher noch nicht beschrieben. Unsere Patientin wurde nach dem AML-BFM-98-Protokoll in modifizierter Form therapiert. Sie befindet sich derzeit in Dauertherapie bei Remission. Unter der durchgeführten Therapie war die Stoffwechseleinstellung weitestgehend zufriedenstellend und es kam zu keiner krisenhaften Verschlechterung des neurologischen Befundes. Die Patientin macht langsame, aber kontinuierliche Entwicklungsfortschritte.

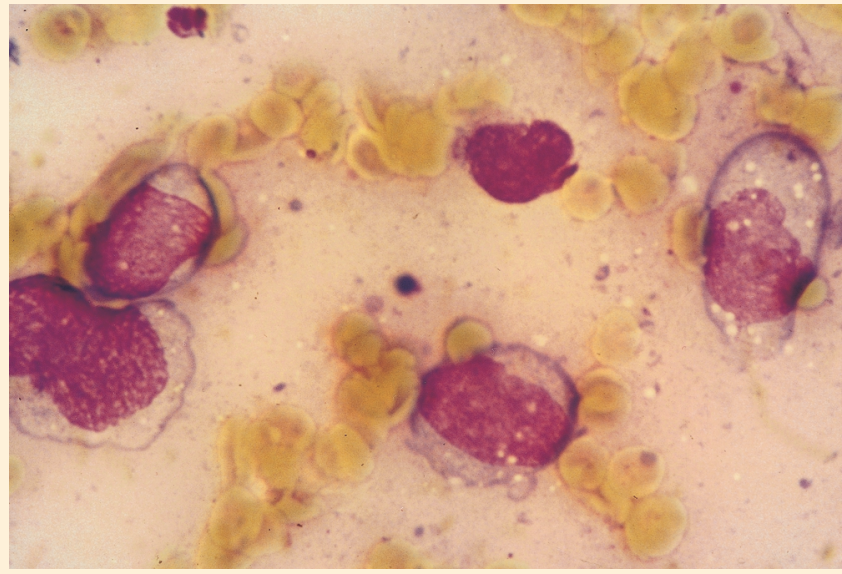

Abb. 3 Nachweis von Blasten im Hautinfiltrat des in Abb. 2 gezeigten Patienten. Das Knochenmark des Patienten (Abb. 4) zeigt ein vergleichbares Bild.

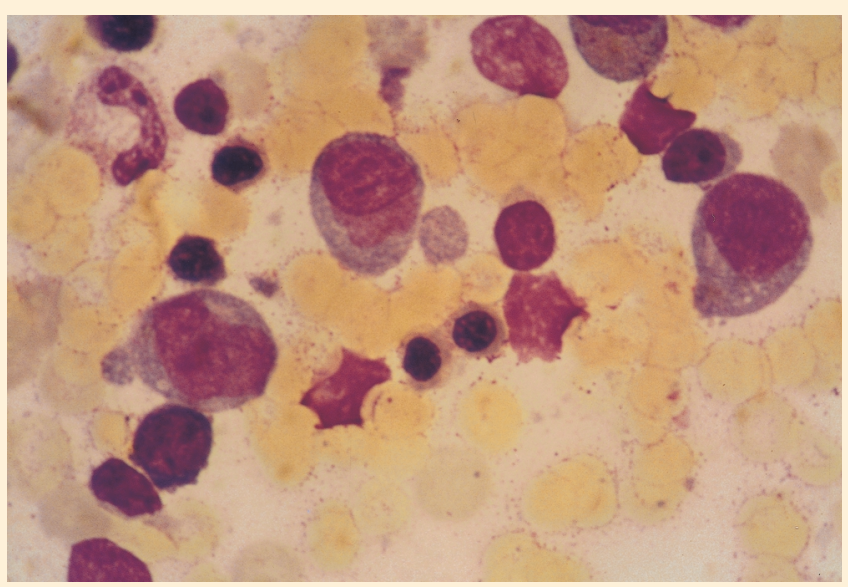

Abb. 4 Knochenmark eines Patienten mit AML. Das histologische Bild entspricht dem Bild des Hautbiopsats.

\section{Literatur}

${ }^{1}$ Desch JK, Smoller BR. The spectrum of cutaneous disease in leukemias. J Cutan Pathol 1993 20: 407-410

2 Molina DN, Sanchez JL, Lugo-Somolinos A. The spectrum of cutaneous lesions in pediatric patients with leukemia. P R Health Sci J 1994 13: 247-249

${ }^{3}$ Baer MR, Barcos M, Farrell H, Raza A, Preisler HD. Acute myelogenous leukemia with leukemia cutis. Eighteen cases seen between 1969 and 1986. Cancer 1989 63: 2192-2200

${ }^{4}$ Sepp N, Radaszkiewicz T, Meijer C], Smolle J, Seewann H, Fritsch P, Kerl H. Specific skin manifestations in acute leukemia with monocytic differentiation. A morphologic and immunohistochemical study of 11 cases. Cancer 1993 71: 124-132

${ }^{5}$ Su WP. Clinical, histopathologic, and immunohistochemical correlations in leukemia cutis-. Semin Dermatol 1994 13: 223-230

${ }^{6}$ Chen MJ, Huang ML, Hung IJ, Kuo TT. Leukemia cutis as the initial manifestation of acute nonlymphocytic leukemia in a young child. Cutis 1997 60: 263-264

${ }^{7}$ Monpoux F, Lacour JP, Hatchuel Y, Hofman P, Raynaud S, Sudaka I, Ortonne JP, Mariani R. Congenital leukemia cutis preceding monoblastic leukemia by 3 months. Pediatr Dermatol 1996 13: 472 - 476

\section{Dr. med. Eva Landmann}

Zentrum für Kinderheilkunde und Jugendmedizin Justus-Liebig-Universität Gießen, Feulgenstraße 12, 35392 Gießen E-mail: Eva.Landmann@paediat.med.uni-giessen.de 